\title{
CONCURRENT ACTIVATION POTENTIATION ENHANCES PERFORMANCE OF SWIMMING RACE START
}

\author{
Vladimir B. Issurin and Oleg Verbitsky \\ Wingate Institute for Physical Education and Sport, Netanya 42902, Israel \\ Technion, Institute of Technology, Haifa 32000, Israel
}

\begin{abstract}
Concurrent activation potentiation (CAP) is recently developed method for enhancing athletic performance in speed-strength exercises. This phenomenon is based on the stimulatory effect of the voluntary contraction of remote muscles, which facilitates activation of prime movers and synergists for targeted exercise. Physiological prerequisites for this ergogenic effect are associated with the integrative function of the cerebral motor cortex and increased excitability of spinal motor neurons. The present study was aimed at evaluating the ergogenic effect producing by CAP treatment on the performance of the race start in competitive swimming. The CAP modified start technique presupposed the teeth clenching and voluntary contraction of abdominal muscles after the preliminary starting command. Eight elite and sub-elite swimmers executed four crawl stroke starts: two CAP modified and two conventional trials with objective registration of start reaction and time to complete a $15-\mathrm{m}$ segment. Statistical analysis included a repeated measures two-way ANOVA test and size effect. Both the p-value and d-value between experimental and control sets were calculated. The average benefit of the CAP modified technique was equal to $0.08 \mathrm{~s}$, which includes an advantage in start reaction equal to $0.05 \mathrm{~s}$. As a result, application of the CAP modified technique to swimming race start resulted in a significant ergogenic effect, which was evaluated by start reaction and performance time on the 15-m segment of distance.
\end{abstract}

Key words: concurrent activation potentiation, ergogenic effect, swimming start, split-unit design 


\section{INTRODUCTION}

The stimulatory effect of concurrent activation of remote muscles on the targeted muscular performance has been known since the late $19^{\text {th }}$ century, when Hungarian physician Erno Jendrassik developed this original technique, termed as the Jendrassik Manoeuver (JM). The JM technique proposes the examination of tendon reflex in the lower limbs when the patient interlocks his/her hands together, pulling them apart and clenching the teeth. Since then the JM has been known as a procedure allowing for potentiating the tendon reflex in neurologically impaired patients [16]. Correspondingly, the JM can be considered as a classic example of the case when remote voluntary contraction (RVC) amplifies the motor output and neural excitability of other muscle groups.

Earlier studies of the potentiation effects of teeth clenching were performed by Japanese researchers, who revealed that teeth clenching produces a significant potentiation effect on shoulder adduction [15] and ankle plantar flexion [13]. The researchers investigated variations of $\mathrm{H}$-reflex following RVC and teeth clenching in a series of in-depth studies. H-reflex, which is evoked by electrical stimulation of the sensory fibers of the innervating nerve, serves as the world-recognized indicator of the excitability of spinal motor neurons; its variables characterize acute and chronic neuromuscular adaptation to maximal effort and resistance training. It has been established that teeth clenching increases the excitability of the soleus H-reflex (i), and the greater force level in teeth clenching produces greater facilitation effect on the soleus H-reflex (ii); facilitation of $\mathrm{H}$-reflex is associated with both the descending influence from the cerebral cortex and the afferent input from the oral-facial region (iii) [10, 11]. The similar facilitation of H-reflex induced by teeth clenching has been found in the forearm muscles [14]. Hiroshi [7] reported that teeth clenching before and during hand griping elicits a significant increase of maximal force and rate of force development as compared with no clenching conditions. Furthermore, a number of studies evaluated the potentiation effect of RVC combined with the performance of various speed-strength exercises, termed Concurrent Activation Potentiation (CAP) [2]. Thus, a significant ergogenic effect of CAP has been found during the performance of the high jump, squat jump, back squat, and knee flexion and extension [3-5]. These publications reported gains of maximal force, rate force development, and jump height that varied within $4.2-32.2 \%$ depending on the character of the variables and experimental conditions.

The possible mechanisms underlying the CAP effects were considered with regards to two major theories attributed to the integrative function of the 
cerebral motor cortex and increased excitability of spinal motor neurons. The first one is focused on intercortical connections between different motor areas of the brain, when activation of one part of the motor cortex that is induced by RVC, such as teeth clenching, affects the activation of neural centers located in another part of the brain. These centers descend a motor command to prime movers, which initiates efforts for targeted exercise [2]. Another theory underlines the role of RVC in the increase of the excitability of spinal motor neurons. This increased excitability is supported by extensive findings of $\mathrm{H}$-reflex investigated during the administration of various RVCs, such as jaw clenching, JM, etc. $[6,13]$. The present study was aimed at evaluating the ergogenic effect produced by the application of CAP to the movement technique of the swimming competitive race start.

\section{MATERIALS AND METHODS}

\section{Subjects}

Eight elite and sub-elite male swimmers took part in the study (mean \pm standard deviation [SD]: age: $19.1 \pm 2.13$ yrs, height: $179.2 \pm 3.2 \mathrm{~cm}$, body mass: $74.3 \pm 3.4 \mathrm{~kg}$, training experience $8.9 \pm 1.3 \mathrm{yrs}$ ). All subjects were sufficiently familiar with the CAP modified start, using this technique in practice and competitions during at least two months. All subjects were informed of the purpose and study design of the present research.

\section{Procedures}

The experimental model of the CAP modified swimming start has been elaborated upon based on the outcomes of previous studies [3-5], and taking into account our experience in start technique investigation in Olympic swimmers [9]. The technique protocol included teeth clenching and voluntary contraction of abdominal muscles, which were initiated immediately after the preliminary starting command ("take your marks"). The time interval between the preliminary command and the starting signal varied between $0.9-1.2 \mathrm{~s}$. This time course completely corresponds to the three-phasic character of the potentiation process: initial teeth clenching and abdominal contraction embraces the $1^{\text {st }}$ phase, whereas the takeoff was corresponded to the $2^{\text {nd }}$ phase, where intensity of the potentiation impact reaches maximum [1]. The subjects were required to stop the teeth clenching immediately after takeoff, while abdominal contraction was maintained according to a habitual coordinative pattern. 
The data were collected during the competitive period. Two VHS video cameras operating at a nominal rate of $50 \mathrm{~Hz}$ were positioned perpendicular to the axis of the swimmers' movement. One camera was placed opposite the start block at $5 \mathrm{~m}$ from the swimmers' location; the second one was installed at the side of the pool and served for measuring time on the mark $15 \mathrm{~m}$. The starting command was produced in accordance with the competition's rules, and a LED signal was transmitted to the videosystem. The videotape data were analyzed with the Ariel Performance Analysis System (Ariel Dynamics Inc., CA, USA); start reaction (SR) was indicated as the time from start signal to takeoff; start efficiency (SE) was assessed with the time to complete the segment $15 \mathrm{~m}$.

\section{Experimental design}

The subjects randomly performed four trials with the crawl stroke, using either the conventional or the CAP modified technique, with the rest interval about $8 \mathrm{~min}$. Eight swimmers were defined as experimental units, while 32 individual measurements, which were performed on eight swimmers, were defined as subunits, correlated the evaluations. A type of start was defined as a repeated treatment design, which was applied to the experimental units. Repeated efforts (trials) within each type of start, defined as a repeated treatment design, were applied to the subunits, correlated observations. Start reaction and start efficiency were defined as a response variable structure. The experimental design structure can be defined as a $2 \times 2$ factorial within-subject repeated experiment with a completely randomized split-unit design structure [8].

\section{Statistical analysis}

In the present study we used a repeated measures two-way ANOVA test with Greenhouse-Geisser epsilon adjustment. Effect size was evaluated by the Cohen's $d$-test for dependent variables. We reported $d$-test value as a measure of effect size, which generally considered 0.20 as a small effect, 0.50 as a medium effect, 0.80 as a large effect, and 1.30 a very large effect. The descriptive statistics were presented by average difference between two sets in $\mathrm{s}$. The statistical significance was established at $\mathrm{p}<0.05$.

\section{RESULTS}

As shown in Table 1, the difference between the experimental and the control sets reached significance for start reaction time $\left(F_{1,7}=20.70, p=0.0029\right)$ and for 
start efficiency $\left(\mathrm{F}_{1,7}=11.137, \mathrm{p}=0.0125\right)$. The start reaction time was shorter in the experimental set as compared with a control set; average difference $=0.05 \mathrm{~s}$, effect size, $\mathrm{d}=1.59$. The start efficiency was higher in experimental set as compared with a control set; average difference $0.08 \mathrm{~s}$, effect size, $\mathrm{d}=1.01$.

Table 1. The effect of start type on the start reaction and start efficiency Repeated Measures two-way ANOVA test

\begin{tabular}{lccccc}
\hline \multirow{2}{*}{ Source of Variance } & df & \multicolumn{2}{c}{ Start reaction } & \multicolumn{2}{c}{ Start efficiency } \\
\cline { 3 - 6 } & & $\mathrm{F}$ & $\mathrm{p}$ & $\mathrm{F}$ & $\mathrm{p}$ \\
\hline Factor A, Control vs. Experiment sets & 1 & 20.070 & 0.0029 & 11.137 & 0.0125 \\
\hline Residual & 7 & & & & \\
\hline Factor B, Trial I vs. Trial II, & 1 & 1.346 & 0.2840 & 0.806 & 0.3990 \\
\hline Residual & 7 & & & & \\
\hline A x B interaction & 1 & 2.944 & 0.1299 & 0.023 & 0.8835 \\
\hline Residual & 7 & & & & \\
\hline Number of total observations & 31 & & & & \\
\hline
\end{tabular}

Note: The statistical significance is established at $p<0.05$.

\section{DISCUSSION}

The study outcomes give strong evidence of the significant ergogenic effect of the CAP application, which was shown both for the start reaction and start efficiency evaluated by time on $15 \mathrm{~m}$. It can be expected that possible effects of CAP treatment may be the increased rate force development in takeoff followed by increased horizontal velocity and flight distance, which may be the major contributors of higher start efficiency. The study revealed one more factor of the ergogenic effect - shortening reaction time. To our knowledge, this is the first evidence that CAP may produce more rapid motor reaction. It is worth noting that the time course of swimming start provides a very small chance for shortening motor reaction, although this possibility was realized in a study where the Grab start was replaced by Track technique [9].

The observations during competitions give additional support for the occurrence of an ergogenic effect during implementation of the CAP modified swim start technique. The monitoring of start reaction is a routine practice of official competitions, where the "Omega" electronic time system presents on-line data and protocols. In our observations, several swimmers employing the CAP start enhanced their reaction time by $0.12-0.16 \mathrm{~s}$ as compared with their habitual data. Of course, these extraordinary gains exceed the range that 
can be expected following neurophysiological and biomechanical prerequisites. However, factors such as cognitive input, beneficial mental concentration, and a more favorable emotional state can also be taken into account as contributors to these motor effects. To date, the swimmers who practiced a CAP modified start have utilized it in European Championships in the event $50 \mathrm{~m}$ backstroke and earned bronze (2011) and gold (2012) medals.

In conclusion, unlike previously conducted studies [e.g., 3-5, 7, 12, 15], the targeted technical component in the present research was not a separate exercise, but rather a relatively short part of the entire athletic performance. Therefore, this study is one of the first attempts to implement CAP treatment into athletic performance that is regulated by official competition protocol. The study outcomes evidence that this implementation is of distinct value and perspective for competitive practice and further research projects in other sports.

\section{REFERENCES}

1. Delwade PJ, Toulouse P. (1981) Facilitation of monosynaptic reflexes by voluntary contraction in remote parts of the body. Mechanisms involved in the Jendrassik maneuver. Brain, 104 (Pt 4), 701-709

2. Ebben WP. (2006) A brief review of concurrent activation potentiation: Theoretical and practical constructs. J Strength Cond Res, 20 (4), 985-991

3. Ebben WP, Flanagan EP, Jenssen RL. (2008) Jaw clenching results in concurrent activation potentiation during the countermovement jump.J Strength Cond Res, $22(6), 1850-54$

4. Ebben WP, Kaufmann CE, Fauth ML. (2010) Kinetic analysis of concurrent activation potentiation during back squats and jump squats. J Strength Cond Res, 24 (6), 1515-19

5. Ebben WP, Petushek EJ, Fauth ML. (2010) EMG analysis of concurrent activation potentiation. Med Sci Sports Exerc, 42 (3), 556-62

6. Gregory JE, Wood SA, Proske U. (2001) An investigation into mechanisms of reflex reinforcement by the Jendrassik maneuver. Exp Brain Res, 138 (3), 366-74

7. Hiroshi C. (2003) Relation between teeth clenching and grip force production characteristics. Kokubyo Gakkal Zasshi, 70 (2), 82-88

8. Hurlbert SH. (2013) Pseudofactorialism, response structures and collective responsibility. Austral Ecology 38: 6, 646-663

9. Issurin VB, Verbitsky O. (2003) Track start vs. Grab start: evidence of the Sydney Olympic games. In: Chatard J-K. (Ed.). Biomechanics and Medicine in Swimming IX. Saint-Etienne University, France, pp. 213-218

10. Miyahara T. (1991) Modulation of soleus H-reflex by teeth clenching. Kokubyo Gakkal Zasshi, 58 (4), 670-686 
11. Miyahara T, Haqiya N, Ohyama T. (1996) Modulation of human soleus H-reflex in association with voluntary clenching of the teeth. J Neurophysiol, 76 (3), 2033-41

12. Sasaki Y, Ueno T, Taniquchi H, Ohyama T. (1998) Effect of teeth clenching on isometric and isokinetic strength of ankle plantar flexion. J Med Dent Sci, 45 (1), 29-37

13. Takada Y, Miyahara T, Tanaka T, Ohyama T, Nakamura Y. (2000) Modulation of H-reflex of pretibial muscles and reciprocal inhibition of soleus muscle during voluntary teeth clenching in humans. J Neurophysiol, 83, 2063-2070

14. Takahashi T, Ueno T, Ohyama T. (2003) Modulation of H-reflex in the forearm during voluntary teeth clenching in humans. Eur J Appl Physiol, 90 (5-6), 651-53

15. Ueno T. (1995) Study on relationship between teeth clenching in intercuspal position and isometric movement of upper limbs. Kokubyo Gakkal Zasshi, 62 (2), 212-253

16. Zehr EP, Stein RB. (1999) Interaction of the Jendrassik maneuver with segmental pre-synaptic inhibition. Exp Brain Res, 124, 474-480

\section{Correspondence to:}

Vladimir B. Issurin,

Wingate Institute of Physical Education and Sport

Netanya 42902

Phone: 972-528470347

Fax: 972 -8639536

e-mail: v.issurin@gmail.com 